TRANSACTIONS OF THE

AMERICAN MATHEMATICAL SOCIETY

Volume 360, Number 12, December 2008, Pages 6181-6195

S 0002-9947(08)04503-0

Article electronically published on July 24, 2008

\title{
BAIRE REFLECTION
}

\author{
STEVO TODORCEVIC AND STUART ZOBLE
}

\begin{abstract}
We study reflection principles involving nonmeager sets and the Baire Property which are consequences of the generic supercompactness of $\omega_{2}$, such as the principle asserting that any point countable Baire space has a stationary set of closed subspaces of weight $\omega_{1}$ which are also Baire spaces. These principles entail the analogous principles of stationary reflection but are incompatible with forcing axioms. Assuming $M M$, there is a Baire metric space in which a club of closed subspaces of weight $\omega_{1}$ are meager in themselves. Unlike stronger forms of Game Reflection, these reflection principles do not decide $\mathrm{CH}$, though they do give $\omega_{2}$ as an upper bound for the size of the continuum.
\end{abstract}

\section{INTRODUCTION AND BASIC THEORY}

For a set $A$ of $\omega$-sequences of ordinals and a set of ordinals $H$ the game $G(A, H)$ has two players who alternate playing ordinals from $H$. Player $I I$ wins if the cooperative play belongs to $A$ and loses otherwise. A weak version of the Game Reflection Principle defined and studied in 9], which we denote $G R P_{\omega}(\theta)$ for an uncountable cardinal $\theta$, asserts that for every $A \subset \theta^{\omega}$, player $I I$ has a winning strategy in $G(A, \theta)$ if and only if $I I$ has a winning strategy in $G(A, H)$ for an $\omega_{1-}$ club of $H \in[\theta]^{\omega_{1}}$. Here an $\omega_{1}$-club means the set of $H$ closed under some function $f: \theta^{<\omega} \rightarrow \theta 1$ The weakened reflection principle obtained by requiring each player to play finite sequences, rather than single ordinals (producing an element of $\theta^{\omega}$ by concatenation of the plays), is immediately equivalent to the Weak Baire Reflection Principle below.

Definition 0.1. $B R P^{w}(\theta)$ asserts that any $A \subset \theta^{\omega}$ is meager if and only if $A \cap H^{\omega}$ is meager in $H^{\omega}$ for an $\omega_{1}$-club of $H \in[\theta]^{\omega_{1}}$.

A stronger version requires reflection of a failure of the Baire Property.

Definition 0.2. $B R P(\theta)$ asserts that any $A \subset \theta^{\omega}$ has the Baire Property in $\theta^{\omega}$ if and only if $A \cap H^{\omega}$ has the Baire Property in $H^{\omega}$ for an $\omega_{1}$-club of $H \in[\theta]^{\omega_{1}}$.

$B R P^{w}$ and $B R P$ will denote the global versions of these principles. Before deducing $B R P$ from Game Reflection we discuss how to expand the scope of these principles to spaces other than the Generalized Baire Spaces (spaces of the form $\left.\theta^{\omega}\right)$. Recall that the weight of a space $X$ is the minimum cardinality of a base for

Received by the editors March 10, 2006.

2000 Mathematics Subject Classification. Primary 03E55; Secondary 03E50.

Key words and phrases. Baire Property, Game Reflection, Martin's Maximum.

${ }^{1}$ In [9] the less restrictive notion of a set closed under $\omega_{1}$-length increasing unions and cofinal in the $\subseteq$-ordering is used. 
the topology of $X$. The point weight of a space $X$ is defined to be the minimum cardinal $\kappa$ such that $X$ has a base $\mathcal{B}$ which satisfies

$$
|\{b \in \mathcal{B} \mid x \in b\}| \leq \kappa
$$

for every $x \in X$. A point countable space is a space with point weight $\omega$. For a space $X$ with base $\mathcal{B}$ and a point $x \in X$ let $\mathcal{B}_{x}=\{b \in \mathcal{B} \mid x \in b\}$. For a set $B \subseteq \mathcal{B}$ let $X_{B}=\left\{x \in X \mid \mathcal{B}_{x} \subseteq B\right\}$. Note that $X_{B}$ is always a closed subspace of $X$. By an $\omega_{1}$-club of subspaces of $X$ of weight $\omega_{1}$ we will mean a set of the form $\left\{X_{B} \mid B \in C\right\}$ for some $\omega_{1}$-club $C \subset[\mathcal{B}]^{\omega_{1}}$. We may thus speak of $B R P(\theta)$ $\left(B R P^{w}(\theta)\right)$ for any class of spaces: for any member space $X$ with weight less than or equal to $\theta$ and $A \subseteq X, A$ has the Baire Property (is meager) in $X$ if and only if $A \cap Y$ has the Baire Property (is meager) in $Y$ for an $\omega_{1}$-club of subspaces $Y \subseteq X$ of weight $\omega_{1}$. It is easy to see that any $\omega_{1}$-club of subspaces of $\lambda^{\omega}$ contains a club of the form $\left\{Y^{\omega} \mid Y \in C\right\}$ for some $\omega_{1}$-club $C \subset[\lambda]^{\omega_{1}}$, so that Baire Reflection as stated in Definitions 0.1 and 0.2 is equivalent to Baire Reflection for the class of generalized Baire spaces.

Theorem 0.3. $G R P_{\omega}(\theta)$ implies $B R P(\theta)$ for completely regular spaces of point weight less than or equal to $\omega_{1}$.

Proof. Fix such a space $X$ of weight $\theta$. Let $\left\{N_{\alpha} \mid \alpha \in \theta\right\}$ enumerate such a base $\mathcal{B}$ for the topology on $X$ with no repetitions, and let $A \subseteq X$ have the reflection property as witnessed by a club $C \subset[\mathcal{B}]^{\omega_{1}}$. A key point is that $C$ can be refined to a club $C_{0} \subset C$ with the property that $b \cap X_{B} \neq \emptyset$ for each $b \in B \in C$. Simply take $C_{0}$ to be the set of $\mathcal{B} \cap H$ in $C$ for an elementary submodel $H \prec H(\kappa)$ with $\omega_{1} \cup\{X, \mathcal{B}\} \subset H$. We describe a game $G\left(A^{*}, \theta\right)$ that simulates the relevant BanachMazur games with player $I I$ as the winner. $A^{*}$ is the set of sequences $\left(\alpha_{i}\right) \in \theta^{\omega}$ such that

(1) $N_{\alpha_{i+1}} \subseteq N_{\alpha_{i}}$ for every $i \in \omega$ and the containment is proper for every $i$ which is nonzero,

(2) if $\alpha_{0}$ is equal to $\alpha_{1}$, then $\bigcap_{i<\omega} N_{\alpha_{i}} \cap A=\emptyset$,

(3) if $\alpha_{0}$ is not equal to $\alpha_{1}$, then $\bigcap_{i<\omega} N_{\alpha_{i}} \subseteq A$.

Now consider an $H \in[\mathcal{B}]^{\omega_{1}}$ in the club $C_{0}$. $H$ is a base for the subspace topology on $X_{H}$. Thus the relevant Banach-Mazur games for $A \cap X_{H}$ are determined. These strategies give rise to a winning strategy for $I I$ in the game $G\left(A^{*}, H^{*}\right)$ in the obvious way where $H^{*}$ is the set of $\alpha$ with $N_{\alpha} \in H$. Thus $I I$ has a winning strategy in $G\left(A^{*}, \theta\right)$ and this in turn gives rise to the Banach-Mazur strategies which show that $A$ has the Baire Property in $X$.

For an infinite cardinal $\kappa$ let $\Gamma_{\kappa}^{U B}$ denote the pointclass of $\kappa$-Universally Baire sets of reals.

Corollary 0.4. $G R P_{\omega}(\kappa)$ implies $\Gamma_{\omega_{1}}^{U B}=\Gamma_{\kappa}^{U B}$. If $\kappa$ is a large enough cardinal (for example a huge cardinal) and $G \subset \operatorname{Col}\left(\omega_{1},<\kappa\right)$ is $V$-generic, then in $V[G]$ every set of reals in $\Gamma_{\omega_{1}}^{U B}$ is determined.

Proof. Game Reflection and hence BRP holds in $V[G]$ by Theorem 0.3 above and Cor. 18 of [9]. The conclusion follows as Universally Baire sets of reals are determined in the presence of two Woodin cardinals (see [4).

We now show that ostensibly stronger versions of $B R P$ and $B R P^{w}$ are equivalent to the versions stated in Definitions 0.1 and 0.2 . Recall that the cellularity of a space 
is the minimum cardinal $\lambda$ such that any pairwise disjoint collection of nonempty open sets has size less than $\lambda$. In a metric space, $\lambda$ is the maximum cardinality of such an antichain. This is because it suffices to consider families of basic open sets, and metric spaces have $\sigma$-discrete bases by Stone's Theorem (see [2]). Let us call a space $X$ a Baire space, or everywhere Baire, if the intersection of any family $\left\{D_{n} \mid n \in \omega\right\}$ of dense open sets is dense. If there is a sequence of open dense sets with empty intersection, then we say that $X$ is meager in iteself.

Theorem 0.5. For any uncountable cardinal $\theta$ the principle $B R P^{w}(\theta)$ is equivalent to its version for metric spaces.

Proof. Assume $B R P^{w}(\theta)$ and let $M$ be a metric space of weight less than or equal to $\theta$. Let $\mathcal{B}$ be a $\sigma$-discrete base for $M$ consisting of $\epsilon$-balls. Suppose $A \subseteq M$ has the reflection property, that is, suppose there is an $\omega_{1}$-club $C \subset[\mathcal{B}]^{\omega_{1}}$ such that $A \cap Y_{B}$ is meager in $Y_{B}$ for any $B \in C$. Fix a maximal pairwise disjoint family $\mathcal{U}$ of elements of $\mathcal{B}$ with the property that every $U \in \mathcal{U}$ is everywhere $\lambda_{U}$ cellular for some cardinal $\lambda_{U}$. By everywhere $\lambda_{U}$ cellular we mean that the cellularity of every nonempty open $V \subseteq U$ is also $\lambda_{U}$. $\mathcal{U}$ can be obtained by collecting the minimal elements of the well-founded relation on $\mathcal{B} \backslash\{\emptyset\}$ defined by $U<V$ if $U \subseteq V$ and $c(U)<c(V)$. Clearly the union of $\mathcal{U}$ is dense in $M$. It suffices to show that $A \cap U$ is meager in $U$ for every $U \in \mathcal{U}$. If $\lambda_{U} \leq \omega_{1}$, then there is $B \in C$ which contains $\{b \in \mathcal{B} \mid b \cap U \neq \emptyset\}$ so that $U \subseteq X_{B}$. It follows that $A \cap U$ is meager in $U$ in this case. Thus we concentrate on $U \in \mathcal{U}$ with $\lambda_{U}>\omega_{1}$. Let $\mathcal{B}_{U}$ denote the set of $b \in \mathcal{B}$ such that $b \subseteq U$. Thus $\mathcal{B}_{U}$ is a base for the subspace topology on $U$. Note that $B_{U}$ has size $\lambda_{U}$. Let $C_{U}=\left\{B \cap \mathcal{B}_{U} \mid B \in C\right\}$. $C_{U}$ is an $\omega_{1}$-club in $\left[\mathcal{B}_{U}\right]^{\omega_{1}}$. Let $U^{*}$ be the metric completion of $U$. We show that there is a dense $G_{\delta}$ set $G \subseteq U^{*}$ and a homeomorphism $\pi: G \rightarrow \lambda_{U}^{\omega}$. Let $\lambda_{U}=\lambda$. For a finite sequence $s \in \lambda^{<\omega}$ let $l h(s)$ denote the length of $s$. For $b \in \mathcal{B}_{U}$ let $b^{*}$ be the extension to $U^{*}$. The set $\mathcal{B}_{U}^{*}=\left\{b^{*} \mid b \in \mathcal{B}_{U}\right\}$ is a base for $U^{*}$. By induction there is a map $\phi: \lambda^{<\omega} \rightarrow \mathcal{B}_{U}$ such that $\phi(\emptyset)=U$ and

(1) $l h(s)=n$ implies $\phi(s)$ has radius less than $\frac{1}{n}$,

(2) $s \subset t$ implies $\phi(t) \subset \phi(s)$,

(3) $\bigcup \phi\left[\lambda^{n}\right]$ is dense in $U$,

(4) $s$ not equal to $t$ implies $c l_{U^{*}}\left(\phi(s)^{*}\right) \cap c l_{U^{*}}\left(\phi(t)^{*}\right)$ is empty.

Thus $G$ defined by

$$
G=\bigcup_{f \in \lambda^{\omega}} \bigcap_{n \in \omega} \phi\left(f\lceil n)^{*}=\bigcap_{n \in \omega} \bigcup_{s \in \lambda^{n}} \phi(s)^{*}\right.
$$

is the desired $G_{\delta}$ with $\pi^{-1}(f)$ defined to be the unique element of $\bigcap_{n \in \omega} \phi(f\lceil n)$. The map $\pi$ acts on elements of $\mathcal{B}_{U}^{*}$ and subsets of $\mathcal{B}_{U}^{*}$. Let $C_{U}^{*}=\left\{B^{*} \mid B \in C\right\}$ where $B^{*}=\left\{b^{*} \mid b \in B\right\}$. Thus $C_{U}^{*}$ is a club in $\left[\mathcal{B}_{U}^{*}\right]^{\omega_{1}}$. Let $C_{U}^{* *}$ be the projection of $C_{U}^{*}$ on $\left\{\phi(s)^{*} \mid s \in \lambda^{<\omega}\right\}=\mathcal{B}_{U}^{* *}$. Thus $\pi\left[C_{U}^{* *}\right]$ is club in $\left[\lambda^{<\omega}\right]^{\omega_{1}}$ (identifying basic open sets in $\lambda^{\omega}$ with elements of $\left.\lambda^{<\omega}\right)$. For $B \in C_{U}$ we have

$$
X_{B^{*}}^{U^{*}} \cap U=X_{B}^{U}
$$

where the superscript indicates the parent space, so that $A$ is meager in $X_{B^{*}}^{U^{*}}$. Thus

$$
X_{B^{*}}^{U^{*}} \cap G=X_{B^{*} \cap \mathcal{B}_{U}^{* *}}^{G}
$$


It follows that $A \cap G$ is meager in $X_{B}^{G}$ for any $B \in C_{U}^{* *}$ so that $\pi[A \cap G]$ is meager in $\pi\left[X_{B}^{G}\right]=X_{\pi[B]}^{\lambda^{\omega}}$. $B R P^{\omega}(\lambda)$ holds as $\lambda \leq \theta$ so $\pi[A \cap G]$ is meager in $\lambda^{\omega}$ and hence $A \cap G$ is meager in $G$. It follows that $A$ is meager in $U$ as desired.

Theorem 0.6. For any uncountable cardinal $\theta, B R P(\theta)$ is equivalent to its version for complete metric spaces.

Proof. Assume $M$ is complete metric and $A \subset M$. Again let $\mathcal{B}$ be a $\sigma$-discrete basis for $M$ consisting of $\epsilon$-balls, and assume $A \cap Y_{B}$ has the Baire Property in $Y_{B}$ for all $B$ in some club $C \subset[\mathcal{B}]^{\omega_{1}}$. Let $\mathcal{U} \subset \mathcal{B}$ be as in the theorem above. Again, it suffices to show that $A \cap U$ has the Baire Property in $U$ for $U \in \mathcal{U}$ with $\lambda_{U}>\omega_{1}$. Since $M$ is already complete, the construction of Theorem 0.5 gives a dense open $G_{U} \subset U$ which is homeomorphic via a map $\pi$ to $\lambda_{U}^{\omega}$ and it suffices to show that $A \cap G_{U}$ has the Baire Property in $G_{U}$. This is deduced by a similar argument as in the theorem above, using $\pi$ and our assumption that $B R P^{w}$ holds in $\lambda_{U}^{\omega}$.

We do not know if $B R P^{w}$ for arbitrary point countable spaces is implied by $B R P^{w}$. We also do not know whether $B R P$ for metric spaces or point countable spaces is a stronger principle than $B R P$. We close this section by defining, in analogy with simultaneous stationary reflection, the Simultaneous Weak Baire Reflection Principle, denoted $B R P_{(2)}^{w}(\theta)$, to assert that pairs of second category sets $X, Y \subset \theta^{\omega}$ reflect to second category sets in a stationary set of closed subspaces $H^{\omega}$ of weight $\omega_{1}$. It will be shown in the next section that $B R P_{(2)}^{w}(\theta)$ implies, and is strictly stronger than, $B R P(\theta)$. Again, nothing more is obtained by expanding the scope of this principle to metric spaces. In analogy with diagonal reflection of stationary sets we have the following principle $B R P_{d}^{w}(\theta)$ which is yet stronger.

Theorem 0.7. Assume $G R P_{\omega}(\theta)$. Suppose $X$ is a completely regular Baire space with a point countable basis. Then a stationary set of closed subspaces $Y \subset X$ of weight $\omega_{1}$ are also Baire spaces.

Proof. This is similar to Theorem 0.3 so we omit some detail. Let $\left\{N_{\alpha} \mid \alpha<\theta\right\}$ enumerate a point countable basis $\mathcal{B}$ for $X$ with no repetitions. Assume to the contrary that there is a club $C \subset[\mathcal{B}]^{\omega_{1}}$ such that $Y_{B}$ is not a Baire space for $B \in C$. Thus there is a function $f: C \rightarrow \theta$ such that $N_{f(B)} \in B$ and $Y_{B} \cap N_{f(B)}$ is meager in itself for every $B \in C$. It is easy to see that an equivalent version of $\operatorname{GRP}_{\omega}(\theta)$ is obtained if player $I I$ always moves first. Let us assume this version and define $A \subset \theta^{\omega}$ to be the set of sequences $\left(\alpha_{n}\right)$ such that $N_{\alpha_{n}}$ is a decreasing chain with empty intersection. For games associated to a $B \in C$, player $I I$ wins by using $f(B)$ as a first move and following the winning strategy for "empty" in the BanachMazur game for $Y_{B} \cap N_{f(B)}$. Thus $I I$ wins the unrestricted game $G(A, \theta)$ with some first move $\alpha$, and it follows that $N_{\alpha}$ is meager in itself, which is a contradiction.

The plan for the rest of the paper is as follows. In Section 2, a relationship with stationary reflection is established and used to show that $B R P^{w}$ does not imply $B R P\left(\omega_{2}\right)$. Throughout, we will strengthen the usual formulation of principles of stationary reflection to assert that reflection occurs stationarily often, where a subset of $[\kappa]^{\omega_{1}}$ is stationary if it meets every $\omega_{1}$-club. These stronger principles follow from Game Reflection as well, though not necessarily from their usual versions (see [3] for more on this issue). One nonstandard principle we use is the following. 
Definition 0.8. $W R P_{(2)}^{c}(\kappa)$ asserts that for any pair of stationary sets $S, T \subset[\kappa]^{\omega}$ whose union contains a club in $[\kappa]^{\omega}$, there is a stationary set of $X \in[\kappa]^{\omega_{1}}$ such that $S \cap[X]^{\omega}$ and $T \cap[X]^{\omega}$ are both stationary in $[X]^{\omega}$.

We will show that $B R P^{w}(\kappa)$ implies $W R P(\kappa)$ and that $B R P(\kappa)$ implies $W R P_{(2)}^{c}(\kappa)$ by associating the space $X(S)=\left\{x \in \kappa^{\omega} \mid \operatorname{ran}(x) \in S\right\}$ to a stationary set $S \subseteq[\kappa]^{\omega}$. Further, we will show that $B R P(\kappa)$ is equivalent to the conjunction of $W R P_{(2)}^{c}(\kappa)$ and $B R P^{w}(\kappa)$. We use this connection to produce a model in which $B R P^{w}$ holds but $B R P\left(\omega_{2}\right)$ does not, namely the model obtained by collapsing a supercompact cardinal to $\omega_{2}$ and then adding a pair of stationary subsets of $\left[\omega_{2}\right]^{\omega}$ which do not simultaneously reflect. In Section 2 we show that $B R P$ holds in the model obtained by the Mitchell collapse of a supercompact (see [11]), which shows that $B R P$ does not imply $C H$, in contrast to König's theorem that $G R P_{\omega}\left(\omega_{2}\right)$ does imply $C H$ (Thm. 8 of [9]). In the last section of this paper we show that Weak Baire Reflection fails under Martin's Maximum in a variety of ways. For example, assuming $M M$, we show that there is a Baire metric space of weight $\omega_{2}$ which has an $\omega_{1}$-club of closed subspaces of weight $\omega_{1}$ which are meager in themselves, and that any set of reals of size $\omega_{1}$ is $\omega_{1}$-Universally Baire but not $\omega_{2}$-Universally Baire.

\section{RELATIONSHIP WITH STATIONARY REFLECTION}

The principle $W R P_{(2)}(\kappa)$ asserts that pairs of stationary sets $S, T \subset[\kappa]^{\omega}$ reflect simultaneously in the sense that there is a stationary set of $X \in[\kappa]^{\omega_{1}}$ such that both $S \cap[X]^{\omega}$ and $T \cap[X]^{\omega}$ are stationary in $[X]^{\omega} . W R P_{(2)}^{c}\left(\omega_{2}\right)$ will only apply to sets $S, T$ whose union contains a club $W R P_{d}(\kappa)$ asserts that for any sequence $\left\{S_{\alpha} \mid \alpha<\kappa\right\}$ of stationary subsets of $[\kappa]^{\omega}$ there is a stationary set of $H \in[\kappa]^{\omega_{1}}$ such that $S_{\alpha} \cap[H]^{\omega}$ is stationary in $H^{\omega}$ for every $\alpha \in H$.

Theorem 1.1. The following implications hold for any uncountable cardinal $\kappa$.

(1) $B R P^{w}(\kappa)$ implies $W R P(\kappa)$.

(2) $B R P(\kappa)$ implies $W R P_{(2)}^{c}(\kappa)$.

(3) $\operatorname{BRP}_{(2)}^{w}(\kappa)$ implies $W R P_{(2)}(\kappa)$.

(4) $B R P_{d}^{w}(\kappa)$ implies $W R P_{d}(\kappa)$.

Proof. For $S \subset[\kappa]^{\omega}$ let $X(S) \subset \kappa^{\omega}$ consist of all sequences $x$ whose range belongs to $S$. The key point is that $X(S)$ is meager if and only if $S$ is nonstationary. For the reverse direction assume $C$ is a club disjoint from $S$ and let $f: \kappa^{<\omega} \rightarrow \kappa$ be such that $C_{f} \subset C$. $f$ gives rise to a winning strategy for $I I$ in the Banach-Mazur game for $\kappa^{\omega} \backslash X(S)$. II simply ensures closure of the range of the final play under the function $f$. For the other direction, assume $I I$ wins the Banach-Mazur game. Let $C$ be the set of $\sigma \in[\kappa]^{\omega}$ such that $\sigma$ is the range of a play by this strategy. Then $C$ is a club disjoint from $S$. The preceding holds just as well in any $H^{\omega}$ with $H$ uncountable and so the implication (1) follows. For (2) let $S$ and $T$ be complimentary mod club and both stationary. Assume there is a club $C \subset[\kappa]^{\omega_{1}}$ such that $S, T$ do not reflect simultaneously at any $H \in C$. Let $X(S) \subset \kappa^{\omega}$ be the space associated to $S$ and let $H \in C$ be arbitrary. Since $S \cap[H]^{\omega}$ either

\footnotetext{
${ }^{2}$ Paul Larson and James Cummings independently pointed out to the second author that $W R P_{(2)}^{c}\left(\omega_{2}\right)$ does not imply $W R P_{(2)}\left(\omega_{2}\right)$; see the remarks following 1.7. Note that nothing is lost by requiring that $S \cup T=[\kappa]^{\omega}$.
} 
contains a club of $[H]^{\omega}$ or is disjoint from one, we conclude that $X(S) \cap H^{\omega}$ is either meager or comeager in $H^{\omega}$ and hence has the Baire Property in $H^{\omega}$. The subspaces $H^{\omega}$ for $H \in C$ contain a club in our topological sense so we conclude that $X(S)$ has the Baire Property in $\kappa^{\omega}$ by $B R P(\kappa)$. If $X(S)$ is comeager in some neighborhood, then $S$ contains a club, and if it is meager, then $S$ is disjoint from a club. In either case, we have a contradiction. (3) is proved similarly. For (4) let $\mathcal{S}=\left\{S_{\alpha} \mid \alpha<\kappa\right\}$ be a sequence of stationary subsets of $[\kappa]^{\omega}$. For $x \in \kappa^{\omega}$ let $\hat{x}$ be defined by $\hat{x}(n)=x(n+1)$. Define the space $X(\mathcal{S})$ to be the set of $x \in \kappa^{\omega}$ such that $\operatorname{ran}(\hat{x}) \in S_{x(0)}$. Thus $X(\mathcal{S})$ is a Baire space by previous remarks. A club of $H \in[\kappa]^{\omega_{1}}$ at which $\mathcal{S}$ does not reflect can be converted into a club of subspaces of $\kappa^{\omega}$ in which the trace of $X(\mathcal{S})$ is not Baire, and so (4) follows.

Theorem 1.2. The following are equivalent.

(1) $B R P^{w}(\kappa)+W R P_{(2)}^{c}\left(\omega_{2}\right)$.

(2) $B R P(\kappa)$.

Proof. Suppose $A \subseteq \kappa^{\omega}$ is such that $A \cap H^{\omega}$ has the Baire Property in $H^{\omega}$ for an $\omega_{1}$-club $C$ of $H \in[\kappa]^{\omega_{1}}$. For $p \in \kappa^{<\omega}$ and $\sigma \in[\kappa]^{\omega}$ let $G_{p}^{* *}(A, \sigma)$ denote the Banach-Mazur game where $I$ and $I I$ alternate playing elements of $\sigma^{<\omega}$ which properly extend each other. I plays first and must extend $p$, and if $x \in \sigma^{\omega}$ is the cooperative play, then $I I$ wins if and only if $x \in A$. For a fixed $p \in \kappa^{<\omega}$ let $S_{i}^{p}$ denote the set of $\sigma \in[\kappa]^{\omega}$ such that player $i$ wins the Banach-Mazur game $G_{p}^{* *}(A, \sigma)$, where $i$ is either $I$ or $I I$. We first claim that $S_{I}^{p} \cup S_{I I}^{p}$ contains a club for any $p \in \kappa^{<\omega}$. Suppose $S=\left[\omega_{2}\right]^{\omega} \backslash\left(S_{I}^{p} \cup S_{I I}^{p}\right)$ is stationary. Then there is $H \in C$ such that $S \cap[H]^{\omega}$ is stationary by $W R P(\kappa)$. Since $A \cap H^{\omega}$ has the Baire Property in $H^{\omega}$ by assumption, there is a winning strategy $\tau$ in the game $G_{p}^{* *}(A, H)$ for one of the players, without loss of generality player $I$, and hence for a club of $\sigma \in[H]^{\omega}$, the restriction $\tau\left\lceil\sigma^{<\omega}\right.$ witnesses $\sigma \in S_{I}^{p}$ giving the desired contradiction. We now show that $S_{I}^{p}$ and $S_{I I}^{p}$ cannot both be stationary. Assume otherwise. By $W R P_{(2)}(\kappa)$ there is $H \in C$ such that both $S_{I}^{p} \cap H^{\omega}$ and $S_{I I}^{p} \cap H^{\omega}$ are stationary in $H^{\omega}$. Either $I$ or $I I$ wins $G^{* *}(A, H)$ via a strategy $\tau$. Any $\sigma$ closed under $\tau$ in the wrong set gives a contradiction. It now follows that for $p \in \kappa^{<\omega}$ either $I I$ wins $G_{p}^{* *}(A, \sigma)$ for a club of $\sigma$ or there is $p \subset q$ such that $I I$ wins $G_{q}^{* *}(\neg A, \sigma)$ for a club of $\sigma$. To see this, fix $p$ and suppose $I I$ fails to win $G_{p}^{* *}(A, \sigma)$ for all $\sigma$ in a stationary set $S$. By intersecting with a club and pressing down we find $q$ so that $I I$ wins $G_{q}^{* *}(\neg A, \sigma)$ for all $\sigma$ in some stationary $\bar{S} \subseteq S$. If this set does not contain a club, then by repeating the previous argument we get an $r$ extending $q$ such that $S_{I}^{r}$ and $S_{I I}^{r}$ are both stationary, contradicting the last claim. It now follows that $A$ has the Baire Property. Suppose $I I$ wins $G_{p}^{* *}(A, \sigma)$ on a club. Then $I I$ wins $G_{p}^{* *}(A, H)$ for an $\omega_{1}$-club and so by $B R P^{w}(\kappa), I I$ must win $G^{* *}(A, \kappa)$.

As a corollary to Theorem 1.1, and the fact that $W R P\left(\omega_{2}\right)$ is equiconsistent with the existence of a weakly compact cardinal, we see that both $B R P^{w}\left(\omega_{2}\right)$ and $B R P\left(\omega_{2}\right)$ are as well. The global principle $B R P^{w}$ is much stronger as it implies the global failure of $\square_{\kappa}$ (see [13]). We now separate $B R P^{w}$ from $B R P\left(\omega_{2}\right)$ using this connection with stationary reflection. The reader is referred to [10 for similar results in the context of $M M$. 
Definition 1.3. $\mathbb{P}$ consists of triples $(p, e, \delta)$ such that

(1) $\delta<\omega_{2}$.

(2) $e: \delta+1 \times \omega_{1} \rightarrow\left[\omega_{2}\right]^{\omega}$.

(3) $\left(e(\gamma, \xi) \mid \xi<\omega_{1}\right)$ is continuous and exhaustive in $[\gamma]^{\omega}$ for each $\gamma \leq \delta$.

(4) If $c f(\gamma)=\omega$, then $e(\gamma, \xi)$ is cofinal in $\gamma$ for each $\xi<\omega_{1}$.

(5) $p: \operatorname{ran}(e) \rightarrow 2$.

(6) For each $\gamma \leq \delta$ the set of $\sigma \in \operatorname{ran}(e)$ such that $p(\sigma)=0$ either contains or is disjoint from a club in $[\gamma]^{\omega}$.

The ordering is reverse inclusion on each coordinate. Note that clause (4) of the definition ensures that $\mathbb{P}$ is $\sigma$-closed.

Lemma 1.4. $\mathbb{P}$ is $\left(\omega_{1}, \infty\right)$-distributive.

Proof. We show that $I I$ wins the game where $I$ and $I I$ alternate playing a decreasing sequence of conditions $\left(p_{\alpha}, e_{\alpha}, \delta_{\alpha}\right)$ for $\alpha<\omega_{1}$ with $I I$ playing on the set of even stages $E$ including limits. At the end, $I I$ must produce a $p$ below every member of the sequence to win. From the existence of a winning strategy for $I I$ one easily deduces that $\mathbb{P}$ is $\left(\omega_{1}, \infty\right)$ distributive (assuming $I$ plays first). Fix $i<2$. Note that $I I$ has complete control over $e_{\gamma}$ at limit stages by clause (4). $I I$ wins by extending arbitrarily at successor stages, and at limits $\alpha$ setting

$$
p_{\alpha}\left(e_{\alpha}\left(\delta_{\alpha}, \xi\right)\right)=i
$$

for every $\xi<\omega_{1}$ and, via some simple bookkeeping, choosing the sequence $\left(e_{\alpha}\left(\delta_{\alpha}, \xi\right) \mid \xi<\omega_{1}\right)$ so that

$$
\left\{e_{\alpha}\left(\delta_{\alpha}, 0\right) \mid \alpha \in E\right\}
$$

is continuous and exhaustive in $[\delta]^{\omega}$ where $\delta$ is the limit of the $\delta_{\alpha}$.

Lemma 1.5. Let $G \subset \mathbb{P}$ be $V$-generic. Suppose $W R P\left(\omega_{2}\right)$ holds in $V[G]$. Then $W R P_{(2)}^{c}\left(\omega_{2}\right)$ fails in $V[G]$.

Proof. The generic filter gives rise to $S^{0}, S^{1} \subset\left[\omega_{1}\right]^{\omega}$ defined by

$$
S^{i}=\bigcup\left\{p^{-1}(i) \mid \exists e, \delta(p, e, \delta) \in G\right\}
$$

for $i<2$ and a set

$$
e=\bigcup\{\operatorname{ran}(e) \mid \exists p, \delta(p, e, \delta) \in G\}
$$

A density argument shows that both $S^{0}$ and $S^{1}$ are stationary and that their union is equal to $e$. By design they do not simultaneously reflect. $e$ contains a club in $\left[\omega_{2}\right]^{\omega}$ by $W R P\left(\omega_{2}\right)$, so the sets $S_{0}, S_{1}$ witness the failure of $W R P_{(2)}^{c}\left(\omega_{2}\right)$ in $V[G]$ as desired.

The following basic result on extending embeddings to generic extensions is attributed to Silver (see [1] or Lemma 1 of [9]).

Lemma 1.6. Suppose $M$ and $N$ are inner models, $k: M \rightarrow N$ is elementary and $\mathbb{P} \in M$. Suppose $\mathbb{P}$ is $M$-generic and $H \subset k(\mathbb{P})$ is $N$-generic. Suppose $k[G] \subset H$. Then there is a unique extension $k^{*}: M[G] \rightarrow N[H]$ of $k$ with $k(G)=H$.

Theorem 1.7. $B R P^{w}$ does not imply $B R P\left(\omega_{2}\right)$. 
Proof. Let $\kappa$ be supercompact and $G \subset \operatorname{Col}\left(\omega_{1},<\kappa\right)$ be $V$-generic. Let $S_{0}, S_{1}$ be $V[G]$-generic subsets of $\left[\omega_{2}\right]^{\omega}$ which do not simultaneously reflect as given by the poset $\mathbb{P}$ described above. If we can show that $B R P^{w}\left(\omega_{2}\right)$ holds in $V[G]\left[S_{0}, S_{1}\right]$, then $B R P\left(\omega_{2}\right)$ must fail in this model by Theorem 1.1 and Lemma 1.5. In fact $B R P^{w}(\theta)$ holds for every $\theta$, but for simplicity let us take $\theta=\omega_{2}$. Let $A \subset \omega_{2}^{\omega}$ have the property that $A \cap[H]^{\omega}$ is meager in $H^{\omega}$ for all $H$ in some club $K \subseteq\left[\omega_{2}\right]^{\omega_{1}}$. Suppose toward a contradiction that $A$ is of second category in $\omega_{2}^{\omega}$. Then one of the sets

$$
A_{0}=\left\{x \in A \mid \operatorname{ran}(x) \in S^{0}\right\}
$$

or

$$
A_{1}=\left\{x \in A \mid \operatorname{ran}(x) \in S^{1}\right\},
$$

say $A_{0}$, is of second category. Let $\mathbb{Q}$ be the poset which shoots a club through $S_{0}$ with conditions of size $\omega_{1}$. More specifically let $p$ and $e$ be the objects given by the generic $\mathbb{P}$ and let $D$ denote the set of $\delta<\omega_{2}$ such that $p(e(\delta, \xi))=0$ for every $\xi<\omega_{1}$. Let $\mathbb{Q}$ be the poset of all continuous successor length sequences from $D$ of size $\omega_{1}$ ordered by reverse inclusion. If $C$ is $V[G]\left[S_{0}, S_{1}\right]$-generic for $\mathbb{Q}$, then $C$ gives rise to a club subset of $S_{0}$. The idea is that the nonmeagerness of $A_{0}$ will be preserved in $V[G]\left[S_{0}, S_{1}\right][C]$ where some generic supercompactness is recovered. Let $(p, \dot{q})$ be a condition in $\operatorname{Col}\left(\omega_{1},<\kappa\right) * \mathbb{P}$ and $\dot{A}$ a term such that $(p, \dot{q})$ forces that $\dot{A}$ is such a set as witnessed by $\dot{K}$ and that $\dot{A}_{0}$ is nonmeager. Let $G^{*} \subset \operatorname{Col}\left(\omega_{1},<j(\kappa)\right)$ be $V$-generic with $p \in G^{*}$ and let $G=G^{*} \cap \operatorname{Col}\left(\omega_{1},<\kappa\right)$. We thus have an embedding $j: V[G] \rightarrow M\left[G^{*}\right]$. In $V\left[G^{*}\right]$ the poset $\mathbb{P} * \mathbb{Q}$ has size $\omega_{1}$. Let $q=\dot{q}_{G} \in \mathbb{P}$. Build a condition $\left(\left(S_{0}, S_{1}\right), C\right)$ in $\mathbb{P} * \mathbb{Q}$ which has a supremum in $j(\mathbb{P} * \mathbb{Q})$ and which meets every dense set in $V[G]$. Now let $\left(S_{0}^{*}, S_{1}^{*}, C^{*}\right)$ be $M\left[G^{*}\right]$-generic for $j(\mathbb{P} * \mathbb{Q})$ below this condition. By Lemma 1.6 above there is a unique extension

$$
j^{*}: V[G]\left[S_{0}, S_{1}\right][C] \rightarrow M\left[G^{*}\right]\left[S_{0}^{*}, S_{1}^{*}\right]\left[C^{*}\right] .
$$

Now we do the usual type of reflection and preservation argument. $A_{0}$ is nonmeager in $V[G]\left[S_{0}, S_{1}\right][C]$. The factor

$$
j(\mathbb{P} * \mathbb{Q}) /\left(S_{0}, S_{1}\right) * C
$$

is $\sigma$-closed and hence $j^{*}[A] \subset j[\kappa]^{\omega}$ is nonmeager in the space $j[\kappa]^{\omega}$ in the model $V\left[G^{*}\right]\left[S_{0}^{*}, S_{1}^{*}\right]\left[C^{*}\right]$ and hence in $M\left[G^{*}\right]\left[S_{0}^{*}, S_{1}^{*}\right]\left[C^{*}\right]$. It follows that the model $M\left[G^{*}\right]\left[S_{0}^{*}, S_{1}^{*}\right]\left[C^{*}\right]$ satisfies the sentence asserting the existence of $H \in[j(\kappa)]^{\omega_{1}}$ with $j^{*}(A) \cap H^{\omega}$ nonmeager in $H^{\omega}$. By elementarity, $V[G]\left[S_{0}, S_{1}\right][C]$ contains such an $H$. Thus $H \in V[G]\left[S_{0}, S_{1}\right]$ as $\mathbb{Q}$ does not add new sets of size $\omega_{1}$, and $H$ must have the property there as well giving the desired contradiction.

Paul Larson noted that the above model can be modified so that $W R P_{(2)}\left(\omega_{2}\right)$ fails but $W R P_{(2)}^{c}\left(\omega_{2}\right)$ holds. Modify the poset $\mathbb{P}$ so that disjoint stationary sets $\left(S^{0}, S^{1}, S^{2}\right)$ are added whose union is $\left[\omega_{2}\right]^{\omega}$ and such that $S^{1}$ and $S^{2}$ do not simultaneously reflect. Suppose $A$ and $B$ are complimentary stationary sets. If $A$ and $B$ both have stationary intersection with some $S^{i}$, then design $\mathbb{Q}$ to shoot a club through $S^{i}$ and use the reflection argument above. Otherwise say $A$ almost contains $S^{0}$ and $B$ almost contains $S^{1}$. Then $A$ and $B$ necessarily reflect simultaneously. Returning to Baire Reflection, we can deduce some corollaries. As a consequence of Theorem 1.2, $B R P_{(2)}^{w}\left(\omega_{2}\right)$ implies $W R P_{(2)}\left(\omega_{2}\right)$ and hence $B R P\left(\omega_{2}\right)$, and in the 
model just described, $B R P\left(\omega_{2}\right)$ holds. Thus $B R P(\theta)$ does not imply $W R P_{(2)}(\theta)$ and hence does not imply $B R P_{(2)}^{w}(\theta)$.

\section{Baire Reflection does not imply $C H$}

König shows in 9 that $G R P_{\omega}\left(\omega_{2}\right)$ implies $C H$. In this section we show that Baire Reflection is consistent with $2^{\omega}=\omega_{2}$. Let $\mathbb{C}_{\kappa}$ be the poset for adding $\kappa$ many Cohen reals. Conditions are finite partial functions from $\kappa \times \omega$ to 2 ordered by reverse inclusion.

Lemma 2.1. Let $\kappa$ and $\delta$ be arbitrary and suppose $G \subset \mathbb{C}_{\kappa}$ is $V$-generic. Suppose $A \subset \delta^{\omega}$ is nonmeager. Then $A$ remains nonmeager in $V[G]$.

Proof. Suppose $\left(\tau^{n} \mid n<\omega\right)$ is a sequence of nowhere dense sets such that

$$
A \subseteq \bigcup_{n<\omega} \tau_{G}^{n}
$$

in $V[G]$ for any $G \subset \mathbb{C}_{\kappa}$ which is $V$-generic. We define a Banach-Mazur strategy for player $I I$ in $V$ as follows. Let $\theta$ be large enough and for $a \in H(\theta)$ let $M_{a}$ be the skolem closure of $a$ inside $H(\theta)$ together with a well-ordering of $H(\theta)$. We will always add the sequence $\left(\tau^{n}\right)$ and $\mathbb{P}$. Fix a bijection $n \rightarrow\left(n_{0}, n_{1}\right)$ from $\omega$ to $\omega \times \omega$. Given a sequence $\left\{s_{0}, s_{1}, \ldots, s_{n}\right\}$ with each $s_{i} \in \delta^{<\omega}$ and $s_{i} \subseteq s_{i+1}$ for each $i$, let $p$ be the $n_{1}^{s t}$ member of $M_{\left\{s_{0}, \ldots, s_{n_{0}}\right\}} \cap \mathbb{P}$ and select $q$ extending $p$ and $s$ extending $s_{n}$ so that $q$ forces that $[s]$ is disjoint from $\tau^{i}$ for each $i \leq n$, where $[s]$ is the basic open neighborhood of $\delta^{\omega}$ determined by $s$. Set $s_{n+1}=s$. Since $I I$ cannot win the Banach-Mazur game for the compliment of $A$ by this strategy there is a play

$$
x=\bigcup\left\{s_{n} \mid n \in \omega\right\}
$$

according to it which belongs to $A$. Let $M$ be the skolem hull of the set of moves of this strategy, and $M_{n}$ the hull of the first $n$ moves. Thus $M=\bigcup_{n<\omega} M_{n}$. There is an $r \in \mathbb{P}$ such that $r$ forces that $x \in \tau^{m}$ for some $m$. Let $p$ be the restriction of $r$ to $M$, and let $k$ be big enough so that $k_{0}=m$ and $p$ is the $k_{1}^{s t}$ member of $M_{\left\{s_{0}, \ldots, s_{m}\right\}}$. Then some extension of $p$ forces that $\left[s_{m+1}\right]$ is disjoint from $\tau^{m}$ and hence that $x \notin \tau^{m}$. Such a condition can be found in $M_{\left\{s_{0}, \ldots, s_{m+1}\right\}}$ so that it is compatible with $r$. This is a contradiction as $x \in\left[s_{m+1}\right]$.

We now briefly exposit (an equivalent version of) the model of 11. For a set $I$ let $\mathbb{C}_{I}$ denote the poset for adding $I$ many Cohen reals. Let $\dot{\sigma}_{I}$ denote the $\mathbb{C}_{I}$ name for the set of all countable partial maps from $I$ to 2 . We work only with full names, that is, we assume that $\tau \in \dot{\sigma}_{I}$ is forced by $\emptyset$ to be such a function. Let $E$ be the set of even ordinals and $O$ the set of odd ordinals. For an ordinal $\kappa$ let $E_{\kappa}=E \cap \kappa$ and $O_{\kappa}=O \cap \kappa$.

Definition 2.2. $\mathbb{M}_{\kappa}$ is the collection of all countable partial maps $p$ such that

(1) $\alpha \in E_{\kappa} \cap \operatorname{dom}(p)$ implies $p(\alpha) \in 2^{<\omega}$,

(2) $\alpha \in O_{\kappa} \cap \operatorname{dom}(p)$ implies $p(\alpha) \in \dot{\sigma}_{E \cap \alpha}$,

(3) $\left\{\alpha \in \operatorname{dom}(p) \cap E_{\kappa} \mid p(\alpha)\right.$ is nonempty $\}$ is finite.

$\mathbb{M}_{\kappa}$ is ordered by setting $p \leq q$ if

(1) $\operatorname{dom}(q) \subseteq \operatorname{dom}(p)$,

(2) $\alpha \in \operatorname{dom}(q) \cap E_{\kappa}$ implies $q(\alpha) \subseteq p(\alpha)$,

(3) $\alpha \in \operatorname{dom}(q) \cap O_{\kappa}$ imples $p\left\lceil E_{\alpha} \Vdash_{\mathbb{C}_{E \cap \alpha}} q(\alpha) \subseteq p(\alpha)\right.$. 
Note the abuse of notation in (3). We are regarding $p\left\lceil I\right.$ as an element of $\mathbb{C}_{I}$ for $I \subset E_{\kappa}$. The following lemma shows that preservation arguments in the context of the Mitchell collapse require only preservation under $\mathbb{C}_{E_{\kappa}}$ and under $\sigma$-closed forcing.

Lemma 2.3. There is a $\sigma$-closed poset $\mathbb{P}_{\kappa}$ and a map $\pi: \mathbb{C}_{E_{\kappa}} \times \mathbb{P}_{\kappa} \rightarrow \mathbb{M}_{\kappa}$ such that whenever $G \subset \mathbb{C}_{E_{\kappa}} \times \mathbb{P}_{\kappa}$ is $V$-generic, then $\pi[G]$ is $V$-generic for $\mathbb{M}_{\kappa}$.

Proof. $\mathbb{P}_{\kappa}$ is the set of $p \in \mathbb{M}_{\kappa}$ which vanishes on even coordinates, that is, such that $\operatorname{dom}(p) \cap E_{\kappa}$ is empty, with the ordering inherited from $\mathbb{M}_{\kappa}$. Clearly $\mathbb{P}_{\kappa}$ is $\sigma$-closed. Define

$$
\pi: \mathbb{C}_{E_{\kappa}} \times \mathbb{P}_{\kappa} \rightarrow \mathbb{M}_{\kappa}
$$

by $\pi(a, p)=a \cup p$. Suppose $D \subset \mathbb{M}_{\kappa}$ is open and dense. Let $(a, p) \in \mathbb{C}_{E_{\kappa}} \times \mathbb{P}_{\kappa}$. Fix a maximal antichain of $b \in \mathbb{C}_{E_{\kappa}}$ below $a$ such that there is $q_{b} \in \mathbb{P}_{\kappa}$ with $b \cup q_{b} \in D$ and $b \cup q_{b} \leq a \cup p$. The conditions $q_{b}$ can be amalgamated (along with $p$ ) into a condition $q$ such that each $b \cup q \in D$ and $q \leq p$ in $\mathbb{P}_{\kappa}$.

Theorem 2.4. Suppose $\kappa$ is supercompact and $G \subset \mathbb{M}_{\kappa}$ is $V$-generic. Then BRP holds in $V[G]$ but $C H$ fails.

Proof. See [10 for a proof that $2^{\omega}=\omega_{2}$ in $V[G]$. Fix $\lambda$ and let $j: V \rightarrow M$ be an embedding with critical point $\kappa$ such that $M^{\theta} \subset M$ for some large enough $\theta$ relative to $\lambda$. Let $G_{j(\kappa)} \subset \mathbb{M}_{j(\kappa)}$ be $V$-generic and $G_{\kappa}=G_{j(\kappa)} \cap \mathbb{M}_{\kappa}$. Thus $j$ extends to

$$
j: V\left[G_{\kappa}\right] \rightarrow M\left[G_{j(\kappa)}\right]
$$

in $V\left[G_{j(\kappa)}\right]$. Since $W R P_{(2)}\left(\omega_{2}\right)$ holds in $V\left[G_{\kappa}\right]$ it suffices, by Theorem 1.2, to show that $B R P^{w}(\lambda)$ holds for any $\lambda$. So suppose $A \in V\left[G_{\kappa}\right]$ and $A \subset \lambda^{\omega}$ is not meager in $\lambda^{\omega}$. We may assume that $A$ is dense. Clearly $j[A]=j(A) \cap j[\lambda]^{\omega}$. Thus, if we can show that $j[A]$ is nonmeager in $j[\lambda]^{\omega}$ in the model $M\left[G_{j(\kappa)}\right]$, then $j[\lambda]$ is the desired element of $[j(\lambda)]^{\omega_{1}}$ and the result follows by reflection and the fact that $j[\lambda]$ is closed under $j(f)$ for any $f: \lambda^{<\omega} \rightarrow \lambda$ in $V\left[G_{\kappa}\right]$. Since $j$ itself is a homeomorphism from $\lambda^{\omega}$ to $j[\lambda]^{\omega}$ which sends $A$ to $j[A], M\left[G_{j(\kappa)}\right] \subset V\left[G_{j(\kappa)}\right]$, and $j\left\lceil\lambda^{\omega} \in V\left[G_{j(\kappa)}\right]\right.$, it suffices to show that $A$ is nonmeager in $V\left[G_{j(\kappa)}\right]$. The key point is that $V\left[G_{j(\kappa)}\right]$ is contained in an extension by a product of $\mathbb{C}_{E_{j(\kappa)}}$ and a $\sigma$-closed poset by Lemma 2.3. It is easy to see that the latter forcing preserves nonmeagerness, and the former is handled by Lemma 2.1. Thus $A$ is nonmeager in an outer model of $V\left[G_{j(\kappa)}\right]$ and hence in $V\left[G_{j(\kappa)}\right]$ as desired.

As a final note, $B R P$ does not imply $2^{\omega_{1}}=\omega_{2}$. The same is true of any consequence of $G R P$.

\section{Failures of Baire Reflection}

Theorem 3.1. Assume $M M$. Then every set of reals A of size $\omega_{1}$ is $\omega_{1}$-Universally Baire but not $\omega_{2}$-Universally Baire.

Proof. Suppose $A$ is a set of reals of size $\omega_{1}$. Let $f: \omega_{1}^{\omega} \rightarrow \omega^{\omega}$ be continuous. Let $O$ be the union of the set of open neighborhoods in $\omega_{1}^{\omega}$ on which $f$ is constant. If $O$ is dense, then we are done. Let $P$ denote the interior of $\omega_{1}^{\omega} \backslash O$. Thus $P$ is nonempty and it suffices to show that $f^{-1}(A) \cap P$ has the Baire Property in $P$. Since $P$ is homeomorphic to $\omega_{1}^{\omega}$ we might as well assume that $f$ is nowhere constant to begin with. Let $B$ be the preimage of $A$ and assume that $B$ does not have the 
Baire Property in $\omega_{1}^{\omega}$. Then there is a nonempty open set (again, which we take to be all of $\omega_{1}^{\omega}$ ) on which $B$ and its complement are everywhere of second category. Consider the ideal

$$
I=\left\{X \subset A \mid f^{-1}(X) \text { is meager in } B\right\} .
$$

$I$ is a $\sigma$-ideal on a set of size $\omega_{1}$ containing all singletons. We claim that $I$ is $\omega_{1}$ dense in the sense that the quotient algebra $P(A) / I$ has a dense set of size $\omega_{1}$. This will be a contradiction by a result in [14] which shows that an $\omega_{1}$-dense ideal on a set of size $\omega_{1}$ cannot exist under $M A_{\omega_{1}}$. Consider an $I$-positive set $X$. By $M A_{\omega_{1}}$ (see [6]) we may write

$$
X=\bigcup_{n<\omega} X_{n},
$$

where each $X_{n}$ is closed in $A$. Thus

$$
f^{-1}(X)=\bigcup_{n<\omega} f^{-1}\left(X_{n}\right),
$$

where each $f^{-1}\left(X_{n}\right)$ is relatively closed in $B$. So there is an $n$ such that the interior of $f^{-1}\left(X_{n}\right)$ is nonempty in the relative topology on $B$, which is to say, there is $s \in \omega_{1}^{<\omega}$ such that

$$
\emptyset \neq[s] \cap B \subset f^{-1}\left(X_{n}\right),
$$

where $[s]=\left\{x \in \omega_{1}^{\omega} \mid s \subseteq f\right\}$. Thus $f[[s] \cap B]$ is an $I$-positive subset of $X$. The claim follows since there are only $\omega_{1}$ such sets.

We now show that $A$ is not $\omega_{2}$-Universally Baire. It is more convenient to regard $A$ as a subset of the unit interval $[0,1]$. We may assume that $A$ is dense. Let $I$ be a precipitous ideal on $A$. For example, simply transfer the nonstationary ideal $N S$ on $\omega_{1}$ to $A$ via a bijection between $\omega_{1}$ and $A$. This ideal is precipitous (in fact saturated) under Martin's Maximum. This means that given any set $X \subset A$ with $X \notin I$ and sequence of $I$ partitions $\left\{W_{n} \mid n \in \omega\right\}$ of $X$ with $W_{n+1}$ a refinement of $W_{n}$, there is a sequence $\left\{X_{n} \mid n \in \omega\right\}$ with nonempty intersection such that each $X_{n}$ is an element of $W_{n}$ (see [7, 8] for more details). Let $\mathcal{B}$ be the set of open subintervals of $[0,1]$ with rational endpoints. We define an operation on the power set of $A$ by

$$
\partial S=S \backslash \bigcup\{b \in \mathcal{B} \mid S \cap b \in I\} .
$$

Note that $S \triangle \partial S \in I$ for any $S \subseteq A$. Define a metric space $M$ as the set of pairs $\left(x,\left(X_{n}\right)\right) \in A \times I^{\omega}$ such that

(1) $x \in \bigcap_{n<\omega} X_{n}$

(2) $\left(X_{n}\right)$ is decreasing modulo $I$,

(3) there are infinitely many $n$ such that $\partial X_{n}=X_{n}$.

$M$ is a subspace of $[0,1] \times I^{\omega}$ with the product topology and is therefore metrizable ( $I$ is discrete). The key use of the precipitous property is the following claim.

Claim 3.2. $M$ is a Baire space.

Proof. Suppose that the empty player wins the Banach-Mazur game on $M$ by a strategy $\tau$. Here empty is the player who moves first and who wins if and only if the intersection of the neighborhoods played is empty. As usual, it suffices to restrict to basic open neighborhoods so we may assume that the output of $\tau$ is always such 
a set. A typical basic open neighborhood in $M$ is of the form $N_{b,\left(X_{i}\right)_{i \leq k}}$, for $b \in \mathcal{B}$ and each $X_{i} \subset b$, defined to be the set of $\left(x,\left(Y_{n}\right)\right) \in M$ such that

(1) $x \in b \cap \bigcap_{i \in \omega} Y_{i}$.

(2) $Y_{i}=X_{i}$ for every $i \leq k$.

Let $\mathcal{N}$ be the set of open neighborhoods as above. For an element $N=N_{b,\left(X_{i}\right)_{i<k}}$ $\in \mathcal{N}$ let $\partial N$ denote $N_{b,\left(X_{i}\right)_{i<k+1}}$ where $X_{k+1}=\partial X_{k}$. Also let $l(N)$ denote $X_{k}$. Let $N$ be the first move according to $\tau$. We build a subtree $T \subset \mathcal{N}^{<\omega}$ as follows. The root of the tree is $N$. Suppose all sequences $t$ of length $k+1$ have been defined. Given such a sequence $t=\left(N_{0}, N_{1}, \ldots, N_{k}\right)$ suppose $N_{k}=N_{b,\left(X_{i}\right)_{i \leq j}}$. By varying plays for $I I$ which extend $\partial N_{k}$ in the Banach-Mazur game and then applying $\tau$, we can produce a set of basic neighborhoods $\mathcal{A} \subset \mathcal{N}$ such that the set of $l(N)$ for $N \in \mathcal{A}$ is a maximal $P(A) / I$ antichain below $l\left(N_{k}\right)$. Sequences of the form $\left(N_{0}, \ldots, N_{k}, N\right)$ for $N \in \mathcal{A}$ will form the successors of $t$ in $T$. For $t \in T$ let $X_{t}=l(N)$ where $N$ is the last neighborhood on the sequence $t$. Let $W_{k}=\left\{X_{t} \mid t \in T\right\}$. Then the partitions $W_{k}$ for $k \in \omega$ witness that the ideal $I$ is not precipitous, giving the desired contradiction.

The projection map

$$
\pi: M \rightarrow A,
$$

which is continuous and nowhere constant, lifts to

$$
\bar{\pi}: \beta M \rightarrow[0,1] .
$$

The preimage $B=\bar{\pi}^{-1}(A)$ has the Baire Property in $\beta M$ by assumption as $\beta M$ has weight $2^{\omega_{1}}=\omega_{2}$. Thus $B$ can be written as the symmetric difference of an open set $U$ and a meager set $F$. As $M$ is a Baire space $M$ is of second category in $\beta M$ and hence $U$ is nonempty as $M \subseteq B$. Let $\left\{D_{n} \mid n \in \omega\right\}$ be open and dense subsets of $U$ such that $\bigcap_{n \in \omega} D_{n} \subset B$. We can now build a Cantor scheme $\left\{U_{s} \mid s \in 2^{<\omega}\right\}$ of open subsets of $U$ such that if $s \in 2^{n}$, then $U_{s} \subset D_{n}$ and for every $s$,

$$
\bar{\pi}\left[\bar{U}_{s 0}\right] \cap \bar{\pi}\left[\bar{U}_{s 1}\right]=\emptyset \text {. }
$$

The set

$$
P=\bigcap_{n} \bigcup_{s \in 2^{n}} \bar{U}_{s}
$$

is perfect in $\beta M$ by compactness and so $\bar{\pi}[P]$ is the desired perfect subset of $A$ by design.

Corollary 3.3. $B R P^{w}\left(\omega_{2}\right)$ fails under $M M$.

Proof. Combine Theorem 3.1 with Theorem 1.2 and the fact that $W R P_{(2)}\left(\omega_{2}\right)$ holds under $M M$.

Theorem 3.4. Assume $M M$. There is a Baire metric space of weight $\omega_{2}$ with a club of subspaces of weight $\omega_{1}$ which are meager in themselves. Hence $B R P^{w}\left(\omega_{2}\right)$ fails.

Proof. For technical reasons we will work in the subspace $M \subset \omega_{2}^{\omega}$ of increasing sequences. $M$ is a complete metric space homeomorphic to $\omega_{2}^{\omega}$. We will produce $X \subset M$ which is everywhere Baire but has the property that $X \cap \delta^{\omega} \cap M$ is meager in $\delta^{\omega} \cap M$ for every $\delta<\omega_{2}$. We will use $M A_{\omega_{1}}$ and $\diamond(S)$ where $S=\left\{\alpha<\omega_{2} \mid c f(\alpha)=\right.$ $\omega\}$. Let $\left(d_{\alpha} \mid \alpha \in S\right)$ be a $\diamond(S)$-sequence. We construct $X=\left\{x_{\alpha} \mid \alpha \in S\right\} \subset M$ as follows. If $d_{\alpha}$ codes a map $\pi_{\alpha}: \alpha^{<\omega} \rightarrow \alpha^{<\omega}$ and an increasing $p \in \omega_{2}^{<\omega}$ with 
the property that $f(s)$ is increasing whenever $s$ is increasing, then we view $\pi_{\alpha}$ as a Banach-Mazur strategy for player $I I$ in $\alpha^{\omega} \cap M$ and let $x_{\alpha} \in M$ be any legal play by this strategy where $I$ has ensured that $x_{\alpha}$ is cofinal in $\alpha$ and extends $p$. Fix a comeager set $D \subseteq M$ and a neighborhood determined by a finite increasing sequence $p$. There is a winning strategy for $I I$ in the game associated to $D, d: \omega_{2}^{<\omega} \rightarrow \omega_{2}$, and there are club many $\alpha<\omega_{2}$ which are closed under this strategy. Thus $d_{\alpha}$ codes $d \uparrow \alpha^{<\omega}$ together with $p$ for some $\alpha \in S$ and hence $x_{\alpha} \in D$ and extends $p$. This shows that $X$ is a Baire space. We claim that $X \cap \delta^{\omega} \cap M$ is meager for every $\delta<\omega_{2}$. If $\delta \in S$ and $\left(\delta_{n} \mid n<\omega\right)$ is a sequence cofinal in $\delta$, then

$$
X \cap \delta^{\omega}=\left\{x_{\delta}\right\} \cup \bigcup_{n<\omega} X \cap \delta_{n}^{\omega} \cap M,
$$

a countable union of sets meager in $\delta^{\omega} \cap M$. So let us assume $\delta$ has cofinality $\omega_{1}$. Here we use $M A_{\omega_{1}}$. Let $\mathbb{P}$ be the set of conditions

$$
p=\left(\Gamma_{p}, X_{p}\right)
$$

such that $\Gamma_{p}$ is a finite subset of $\delta$ and $X_{p}$ is a finite subset of $S \cap \delta$. For a pair of conditions $p, q$ we define $p \leq q$ if $\Gamma_{q} \subseteq \Gamma_{p}, X_{q} \subseteq X_{p}$ and for every $\alpha \in X_{q}$ we have $\left(\Gamma_{p} \backslash \Gamma_{q}\right) \cap \operatorname{ran}\left(x_{\alpha}\right)=\emptyset$.

Claim 3.5. $\mathbb{P}$ satisfies the countable chain condition.

Proof. Suppose $\left(p_{\alpha} \mid \alpha<\omega_{1}\right)$ is an antichain. By two applications of the $\Delta$-system lemma we may assume that there are sets $s$ and $t$ such that

$$
\Gamma_{p_{\alpha}} \cap \Gamma_{p_{\beta}}=s \text { and } X_{p_{\alpha}} \cap X_{p_{\beta}}=t
$$

for every $\alpha<\beta<\omega_{1}$. There is a subsequence which we reindex as

$$
\left(p_{\alpha} \mid \alpha<\omega^{2}+1\right)
$$

such that for every $\alpha<\beta<\omega^{2}$,

$$
\max \left(\Gamma_{p_{\alpha}} \backslash s\right)<\min \left(X_{p_{\beta}} \backslash t\right) .
$$

It now follows that some $x_{\gamma}$ with $\gamma \in X_{p_{\omega^{2}+1}}$ has order type greater than $\omega$ which is a contradiction.

If $G \subset \mathbb{P}$ is a filter and $G$ meets the requisite dense sets, then

$$
\Gamma=\bigcup\left\{\Gamma_{p} \mid p \in G\right\}
$$

is an unbounded subset of $\delta$ which is almost disjoint from $x_{\alpha}$ for every $\alpha \in S \cap \delta$. An easy argument shows that each

$$
X_{k}=\left\{x_{\alpha} \mid \alpha \in S \cap \delta \text { and } \forall n>k x_{\alpha}(n) \in \delta \backslash \Gamma\right\}
$$

is meager, from which it follows that $X \cap \delta^{\omega} \cap M$ is meager in $\delta^{\omega} \cap M$.

Theorem 3.6. $B R P^{w}\left(2^{\omega}\right)$ for point countable spaces fails under $M A_{\omega_{1}}$.

Proof. We will use the tree $\sigma \mathbb{Q}$ of countable well-ordered increasing sequences of rationals which are bounded. We do not require the sequences to be continuous. For $t \in \sigma \mathbb{Q}$ and $q \in \mathbb{Q}$ let $B_{t, q}$ denote

$$
\{s \in \sigma \mathbb{Q} \mid t \subseteq s \text { and } \sup (s)<q\} .
$$

These sets together with their complements form a point countable base for a zero-dimensional topology on $\sigma \mathbb{Q}$. We claim that $\sigma \mathbb{Q}$ is a Baire space. In the 
relevant Banach-Mazur game, the nonempty player wins by ensuring that the $t_{n}$ are increasing, where $\left\{B_{t_{n}, q_{n}} \mid n<\omega\right\}$ is the sequence of plays, in which case

$$
\bigcup_{n<\omega} s_{n} \in \bigcap_{n<\omega} B_{t_{n}, q_{n}} .
$$

An $\omega_{1}$-club of closed subspaces of $\sigma \mathbb{Q}$ are subtrees of $\sigma \mathbb{Q}$ (viewed as a tree in the obvious way), and have no isolated points. Simply close under the successor and predecessor function. Any such $T$ has size $\omega_{1}$ and hence is special under $M A_{\omega_{1}}$ (see [16] for example). Let $T$ be such a subspace and $\pi: T \rightarrow \omega$ a specializing map. Each $\pi^{-1}(n)$ is closed and nowhere dense in $T$. Thus $T$ is meager in itself.

We conclude with some observations concerning reflection of second category to subspaces of countable weight. The principle analogous to $B R P^{w}(\kappa)$ would assert that $X \subset \kappa^{\omega}$ is meager iff $X \cap \sigma^{\omega}$ is meager for a club of $\sigma \in[\kappa]^{\omega}$. In the case $\kappa=\omega_{1}$ a counterexample exists under $\diamond$, and we suspect that the principle is true under the Proper Forcing Axiom, though we do not have a proof at present. For $\kappa=\omega_{2}$, the principle is outright false.

Theorem 3.7. There is $X \subset \omega_{2}^{\omega}$ of second category such that $X \cap \sigma^{\omega}$ is meager in $\sigma^{\omega}$ for a club of $\sigma \in\left[\omega_{2}\right]^{\omega}$.

Proof. Fix a sequence $\left\{C_{\alpha} \mid \alpha<\omega_{1}\right.$ and $\left.c f(\alpha)=\omega\right\}$ with each $C_{\alpha}: \omega \rightarrow \alpha$ cofinal and increasing, having the property that every club subset of $\omega_{2}$ includes the range of one (and hence stationarily many) $C_{\alpha}$ (see [12]). Fix a surjective map $\phi:[\omega]^{\omega} \rightarrow\left[\omega_{1}\right]^{\omega}$. A real $r \in 2^{\omega}$ codes a sequence of reals $\left\{r_{n} \mid n<\omega\right\}$ defined by $r_{n}(k)=r\left(2^{n}(2 k+1)\right)$. We associate another operation $\phi^{*}:[\omega]^{\omega} \rightarrow\left[\omega_{1}\right]^{\omega}$ defined by

$$
\phi^{*}(r)=\bigcup_{n<\omega} \phi\left[r_{n}\right]
$$

where we identify a real $s \in 2^{\omega}$ with $s^{-1}(1) \in[\omega]^{\omega}$. For $\sigma \in\left[\omega_{2}\right]^{\omega}$ define $\nu_{\sigma}$ by

$$
\nu_{\sigma}=\pi_{\sigma}^{-1}\left(\phi^{*}(\operatorname{osc}(\sigma))\right)
$$

where $\pi_{\sigma}: \sigma \rightarrow o t p(\sigma)$ is the collapse of $\sigma$ and $\operatorname{osc}(\sigma)$ is defined to be the set

$$
\left\{n \mid \sigma \cap\left[C_{\text {sup }(\sigma)}(n), C_{\sup (\sigma)}(n)\right) \neq \emptyset\right\} .
$$

The sequence $\left\{\nu_{\sigma} \mid \sigma \in\left[\omega_{2}\right]^{\omega}\right\}$ can be shown to have the following property: for any $X \subset \omega_{2}$ the set of $\sigma \in\left[\omega_{2}\right]^{\omega}$ such that $X \cap \sigma=\nu_{\sigma}$ is stationary in $\left[\omega_{2}\right]^{\omega}$ (see Lemma 43 of [15] and [5]). We may view $\nu_{\sigma}$ as coding a comeager subset of $\sigma^{\omega}$ (as in Theorem 3.4) in some canonical way. For example, fix a bijection

$$
c: \omega_{2} \times \omega_{2}^{<\omega} \rightarrow \omega_{2},
$$

and observe that if $Y \subset \omega \times \omega_{2}^{<\omega}$ is a comeager set in the obvious way ( $\hat{Y}=\{x \in$ $\omega_{2}^{\omega} \mid \forall n \exists k(n, x\lceil k) \in Y\}$ is comeager), then $c^{-1}(c(Y) \cap \sigma)=\hat{Y} \cap \omega \times \sigma^{<\omega}$ is comeager in $\sigma^{\omega}$ for a club of $\sigma \in\left[\omega_{2}\right]^{\omega}$. Define the space $X \subset \omega_{2}^{\omega}$ to be $\left\{x_{\sigma} \mid \sigma \in\right.$ $\left[\omega_{2}\right]^{\omega}$ where $x_{\sigma}$ is an element of $c^{-1}\left(\nu_{\sigma}\right)$ in the case that this is a code for a comeager set in $\sigma^{\omega}$, and such that the range of $x_{\sigma}=\sigma$. It is clear that $X$ is of second category as for any comeager set $Y \subset \omega_{2}^{\omega}$ there is $\sigma$ such that $c^{-1}\left(\nu_{\sigma}\right)=Y \cap\left(\omega \times \sigma^{<\omega}\right)$ so that $x_{\sigma}$ is in $\hat{Y}$. On the other hand, $X \cap \sigma^{\omega}$ is a singleton (or empty), and hence meager, for every $\sigma \in\left[\omega_{2}\right]^{\omega}$. 


\section{REFERENCES}

[1] Cummings, J., Large Cardinal Properties of Small Cardinals, 1988, unpublished manuscript

[2] Engelking, R., General Topology, Heldermann Verlag, 1989 MR.1039321 (91c:54001)

[3] Feng, Q., Jech, T., Local Clubs, Reflection, and Preserving Stationary Sets, Proceedings of the London Mathematical Society, Vol. 58, 1989, 237-257 MR977476 (90a:03072)

[4] Feng, Q., Magidor, M., Woodin, H., Universally Baire Sets of Reals, MSRI Publications 26, 1992 MR.1233821 (94g:03095)

[5] Foreman, M., Todorcevic, S., A New Löwenheim-Skolem Theorem, Transactions of the American Mathematical Society 357, 2005, 1693-1715 MR2115072 (2005m:03064)

[6] Fremlin, D.H., Consequences of Martin's Axiom, Cambridge University Press, 1984 MR780933 (86i:03001)

[7] Galvin, F., Jech, T., Magidor, M., An Ideal Game, Journal of Symbolic Logic, Vol. 43, No. 2, 1978, 284-292 MR0485391 (58:5237)

[8] Jech, T., Magidor, M., Mitchell, M., Prikry, K., Precipitous Ideals, Journal of Symbolic Logic, Vol. 45, No. 1, 1980, 1-8 MR560220 (81h:03097)

[9] König, B., Generic Compactness Reformulated, Archive for Mathematical Logic 43, 2004, no. 3, 311-326 MR 2052885 (2005f:03079)

[10] Larson, P., Separating Stationary Reflection Principles, Journal of Symbolic Logic, Vol. 65, No. 1, 2000, 247-258 MR1782117 (2001k:03094)

[11] Mitchell, W., Aronsajn Trees and the Independence of the Transfer Property, Annals of Mathematical Logic, Vol. 5, No. 1, 1975, 21-46 MR0313057 (47:1612)

[12] Shelah, S., Cardinal Arithmetic, Vol. 29 of Oxford Logic Guides, Oxford University Press, 1994 MR.1318912 (96e:03001)

[13] Steel, J., PFA implies $A D^{L(\mathbb{R})}$, Journal of Symbolic Logic, Vol. 45, No. 4, 2005, 1255-1296 MR:2194247

[14] Taylor, A.D., Regularity Properties of Ideals and Ultrafilters, Annals of Mathematical Logic 16, 1979, No. 1, 33-55 MR530430 (83b:04003)

[15] Todorcevic, S., Localized Reflection and Fragments of PFA, DIMACS Series in Discrete Mathematics and Theoretical Computer Science, vol. 58, 2002, 135-148 MR1903856 (2003g:03081)

[16] Todorcevic, S., Trees and Linearly Ordered Sets, Handbook of Set Theoretic Topology, North Holland, 1984, 235-293 MR776625 (86h:54040)

Department of Mathematics, University of Toronto, Toronto, Canada M5S 2E4 and - Universite Paris 7-CNRS, UMR 7056, 2 Place Jussieu, 75251 Paris Cedex 05, France

E-mail address: stevo@math.toronto.edu

Department of Mathematics, University of Toronto, Toronto, Canada M5S 2E4

Current address: Department of Mathematics, Wesleyan University, 265 Church Street, Middletown, Connecticut 06459-0128

E-mail address: azoble@wesleyan.edu 